\title{
Stability study of nasal powder formulation containing nanosized lamotrigine
}

\author{
PÉTER GIESZINGER, GÁBOR KATONA, PIROSKA SZABÓ-RÉVÉSZ, RITA AMBRUS*
}

Institute of Pharmaceutical Technology and Regulatory Affairs, University of Szeged, Faculty of Pharmacy, Szeged, Hungary

\author{
*Corresponding author: Rita Ambrus \\ E-mail: arita@pharm.u-szeged.hu
}

Received: 7 April 2020 / Revised: 27 May 2020 / Accepted: 27 May 2020

\begin{abstract}
Drug administration through the nose offers great possibilities which have been discovered in the past few decades. Besides the most known local effect, systemic and central nervous system effect is also available, the administration is non-painful and the degradation effect of the gastrointestinal tract can be avoided. Amongst the nasal formulations, powders have become more popular as their stability is favorable compared to the liquid formulations and a higher doses can be administered in powder form. The quality insurance and stability of the products in the pharmaceutical field have gained considerable attention in the last decades. Due to this fact, the aim was to execute a long-term stability study of a previously developed, nanosized lamotrigine (LAM) containing nasal powder (NP) formulation. The results of the stability test showed that the NP formulation preserved its key properties (particle size, morphology, structure and in vitro drug release) after 6 months of storage.
\end{abstract}

Keywords: nasal delivery, stability test, nanotechnology, nasal powder, lamotrigine

\section{Introduction}

The nose offers a great possibility to avoid adverse events and increase patient compliance[1-4]. Due to its advantageous properties local, systemic and central nervous (CNS) system effects are also available [5-7]. The application of innovative and efficient products, that are containing nanoparticles, may lead to the improvement of different therapies $[8,9]$. The quality insurance of pharmaceutical products has received considerable attention in the past few years. That is why the stability of the formulations has become extremely important and therefore, quality influencing parameters need to be kept constant during the transport, storage, and application. Generally, solid dosage forms (e.g. nasal powders) have better stability than liquid formulations [10-12].

In our previous studies, a nanosized lamotrigine (LAM) containing nasal powder (NP) product was researched and developed. The investigations of the product showed that due to the effect of milling, the nanosized LAM particles became partly amorphous and distributed homogenously on the polymer, polyvinyl alcohol (PVA) matrix. Moreover, the product performed rapid and high amount of drug release in vitro and in vivo. This study aimed to carry out the long-term stability study of the previously developed NP formulation, which contained nanosized LAM [13-15].

\section{Materials and methods}

\subsection{Materials}

Lamotrigine, poorly water-soluble $(0.17 \mathrm{mg} / \mathrm{mL}$ at $25^{\circ} \mathrm{C}$ ) was purchased from Teva Ltd. (Budapest, Hungary). PVA ( $\mathrm{Mw}=27,000)$, water-soluble synthetic polymer - that was applied to stabilize the unique drug particles, thus improving their absorption - was supplied by ISP Customer Service GmBH (Cologne, Germany).

\subsection{Sample Preparation}

PVA was used as an additive during the sample preparation process to maintain the stability and individuality of LAM particles. NP sample was produced as follows: $0.8 \mathrm{~g}$ PVA and $1 \mathrm{~g}$ LAM were mixed in a Turbula mixer (Turbula System Schatz; Willy A. Bachofen AG Maschinenfabrik, Basel, Switzerland) using $60 \mathrm{rpm}$ for $10 \mathrm{~min}$. After mixing, the sample was placed into a planetary ball mill (Retsch PM 100; Retsch, Neuhausen, Germa- 
ny) and milled in a $50 \mathrm{~mL}$ capacity milling chamber for $1.5 \mathrm{~h}$ on $400 \mathrm{rpm}$ with 10 steel balls (diameter $10 \mathrm{~mm}$, the weight of each ball $4.02 \mathrm{~g}$ ). In the case of the physical mixture (PM), PVA was milled for $1.5 \mathrm{~h}$ on $400 \mathrm{rpm}$ and then-according to our previous experiments-it was mixed with unmilled LAM using the same Turbula mixer for 10 min on $60 \mathrm{rpm}$.

\subsection{Circumstances of the stability}

Stability tests were performed according to the ICH Q1A guideline [16] in Binder KBF 240 (Binder $\mathrm{GmbH}$, Tuttlingen, Germany) equipment, with a constant-climate chamber. An electronically controlled APT.line ${ }^{\mathrm{TM}}$ line preheating chamber and refrigerating system ensured temperature accuracy and reproducibility of the results in the temperature range between 10 and $70{ }^{\circ} \mathrm{C}$ and the relative humidity (RH) range between 10 and $80 \%$. The stability test was performed at $25 \pm 2{ }^{\circ} \mathrm{C}$ with $50 \pm 5 \% \mathrm{RH}$ (room conditions). Samples were kept in a closed jar during the tested period. Sampling was carried out after 1 day; 3 and 6 months.

\subsection{Particle size and morphology characterization}

The particle size of the microparticles was characterized by using Leica Image Processing and Analysis System device (Leica Q500MC; Leica Microsystems, Wetzlar, Germany). The test parameters of 300 particles were their length, width, area, and district/convex perimeter.

The morphology and the size the LAM nanoparticles - that were on the surface of the polymer microparticles - were investigated by SEM (Hitachi S4700; Hitachi Ltd., Tokyo, Japan) at $10 \mathrm{kV}$. The samples were gold-palladium-coated (90 s) with a sputter coater (Bio-Rad SC502; VG Microtech, Uckfield, UK) using an electric potential of $2.0 \mathrm{kV}$ at $10 \mathrm{~mA}$ for $10 \mathrm{~min}$. The air pressure was 1.3-13.0 mPa. Distribution of LAM particle diameter was obtained by analyzing SEM images with the ImageJ software (1.50i; Java 1.6.0_20 [32-bit]; Windows NT) environment using approximately 500 particles [17].

Statistical analysis was performed with TIBCO Statistica ${ }^{\circledR}$ 13.4 (Statsoft Hungary, Budapest, Hungary). All reported data are means \pm SD. The Student's t-test was used to determine the statistical significance. Changes were considered statistically significant at $\mathrm{p}<0.05$.

\subsection{Structural investigations}

\subsubsection{Thermoanalytical measurements}

The Mettler Toledo TGA/DSC 1 thermal analysis system (Mettler-Toledo GmbH, Greifensee, Switzerland) was applied to characterize the structures of the products. The DSC (differential scanning calorimetry) and TG (thermogravimetry) measurements were made by examining approximately 3-5 $\mathrm{mg}$ of samples in the temperature range between $25^{\circ} \mathrm{C}$ and $230{ }^{\circ} \mathrm{C}$ at a heating rate of $5{ }^{\circ} \mathrm{C} / \mathrm{min}$ under constant argon flow of $150 \mathrm{~mL} / \mathrm{min}$ and nitrogen flow of $50 \mathrm{~mL} / \mathrm{min}$. Data analysis was performed using the STARe software (Mettler-Toledo $\mathrm{GmbH}$, Greifensee, Switzerland). The cristallinity indexes were calculated based on the ratio Normalized integrals, where the PM samples was considered as $100 \%$.

\subsubsection{X-ray powder diffraction (XRPD)}

The XRPD measurement was carried out with a BRUKER D8 advance X-ray powder diffractometer (Bruker AXS GmbH, Karlsruhe, Germany) with $\mathrm{Cu} \mathrm{K} \lambda \mathrm{I}$ radiation $(\lambda=1.5406 \AA)$ and a VÅNTEC-1 detector (Bruker AXS GmbH, Karlsruhe, Germany). The powder samples were loaded in contact with a plane quartz glass sample slide with an etched square and measured. Samples were scanned at $40 \mathrm{kV}$ and $40 \mathrm{~mA}$. The angular range was $3^{\circ}-40^{\circ} 2 \theta$, at a step time of 0.1 seconds and a step size of $0.007^{\circ}$. All manipulations, including $\mathrm{K} \alpha 2$ stripping, background removal and smoothing of the area under the peaks of the diffractograms, were performed using the DIFFRACplus EVA software. The crystallinity index $\left(X_{C}\right)$ values were calculated based on the following formule, where A marks the area under the whole curve:

$$
\mathrm{X}_{\mathrm{c}}=\mathrm{A}_{\text {crystalline }} /\left(\mathrm{A}_{\text {crystalline }}+\mathrm{A}_{\text {amorphous }}\right) * 100
$$

PM sample was considered as 100\%.

\subsection{In vitro release study}

The modified paddle method (USP dissolution apparatus, type II; Pharma Test, Hainburg, Germany) was used to examine the dissolution rate of LAM-containing co-milled nasal powders and determine the drug release profile from the samples. The test was carried out under nasal conditions 
Table 1 The results of particle size investigation of the product.

\begin{tabular}{c|c|c|c|c}
\hline & Average size of the product $(\boldsymbol{\mu m})$ & $\mathbf{t}$-value & p-value & Significance \\
\hline 1-day & $29.91 \pm 15.85$ & -0.1435 & 0.8883 & n.s. \\
\hline 3 months & $28.48 \pm 12.81$ & 0.2994 & 0.7690 & n.s. \\
\hline 6 months & $26.52 \pm 11.14$ & 0.9064 & 0.3801 & n.s. \\
\hline
\end{tabular}

In the table n.s means that there is no significant difference at $95 \%$ level.

Table 2 The results of particle size investigation of LAM.

\begin{tabular}{c|c|c|c|c}
\hline & Average size of LAM (nm) & $\mathbf{t}$-value & p-value & Significance \\
\hline 1-day & $97 \pm 60$ & 1.2382 & 0.2347 & n.s. \\
\hline 3 months & $105 \pm 77$ & 0.7934 & 0.4408 & n.s. \\
\hline 6 months & $120 \pm 84$ & -0.0408 & 0.9687 & n.s. \\
\hline
\end{tabular}

In the table n.s means that there is no significant difference at $95 \%$ level.

for temperature and $\mathrm{pH} .100 \mathrm{ml}$ phosphate-buffered saline solution (PBS of $\mathrm{pH} 5.60$ at $30{ }^{\circ} \mathrm{C}$ ) was used as a medium in $150 \mathrm{~mL}$ vessels, in which 108 $\mathrm{mg}$ of the samples were tested. The paddle was placed to halfway through the medium and was rotated at $50 \mathrm{rpm}$, and the sampling points were at $5 \mathrm{~min}, 10 \mathrm{~min}, 15 \mathrm{~min}, 30 \mathrm{~min}, 45 \mathrm{~min}$, and $60 \mathrm{~min}$. In each sampling point $2 \mathrm{~mL}$ samples were taken, which volume was immediately replaced with fresh buffer solution to maintain the permanent volume. Cellulose ester membranes with $0.45 \mu \mathrm{m}$ pore diameter was used for filtration. The sampling points were more frequent in the beginning of the investigation as the mucociliary clearance renews the mucus every $15 \mathrm{~min}$. The following sampling points offered extra information about the dissolution behavior of LAM. After filtration, the drug content of the aliquots was determined using spectrophotometry (Unicam UV/VIS Spectrophotometer) at $307 \mathrm{~nm}$. The tests were carried out in triplicates.

\section{Results and discussion}

\subsection{Characterization of particle size and morphology}

The results of particle size determination resulted in same particle size of the product during the examined period (Table 1), which shows that the product's particles did not aggregate. The size falls into the range which is desired in the case of nasal powders, which is $10-40 \mu \mathrm{m}$ [17].

The particle size of LAM in the formulation showed an increase with relatively high standard deviation, which is related to the nanoscale range and the ImageJ technique used (Table 2). However, according to results of the statistical analysis there is no significant difference in the particle sizes during the storage period, thus the previously experienced rapid and high degree of release can be predicted.

The SEM pictures confirm the results of the particle size determination as the nanosized LAM particles can be seen on the PVA surface in a homogenous distribution and showed no sign of aggregation during the tested period (Figure 1).

\subsection{Structural investigations}

The DSC curves (Figure 2A) in the tested period shows that the partly amorphous property of the sample did not change considerably. The melting point of LAM was between $216{ }^{\circ} \mathrm{C}$ and $217{ }^{\circ} \mathrm{C}$, which became lower $\left(\sim 204^{\circ} \mathrm{C}\right)$ due to the effect of milling and the presence of PVA. The two melting points merged as LAM melted on $\sim 204{ }^{\circ} \mathrm{C}$, while PVA did it on $\sim 210{ }^{\circ} \mathrm{C}$. However, the character and area of the endothermic peak are almost the same in each sampling point in the case of the NP formulation. Compared to the PM, which was regarded as $100 \%$, the crystallinity degrees of the samples were between 42 and $48 \%$.

This immutability can be seen in the case of XRPD diffractograms (Figure 2B). According to the
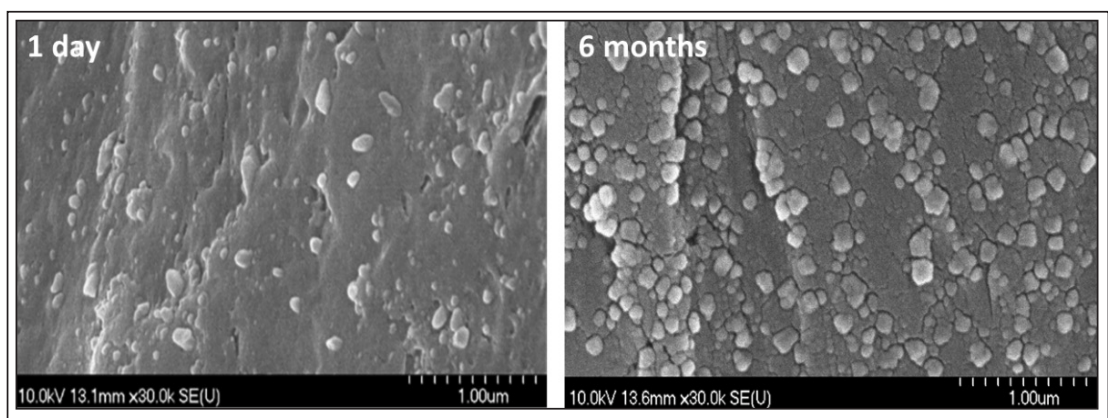

Figure 1 The SEM pictures of the NP samples 


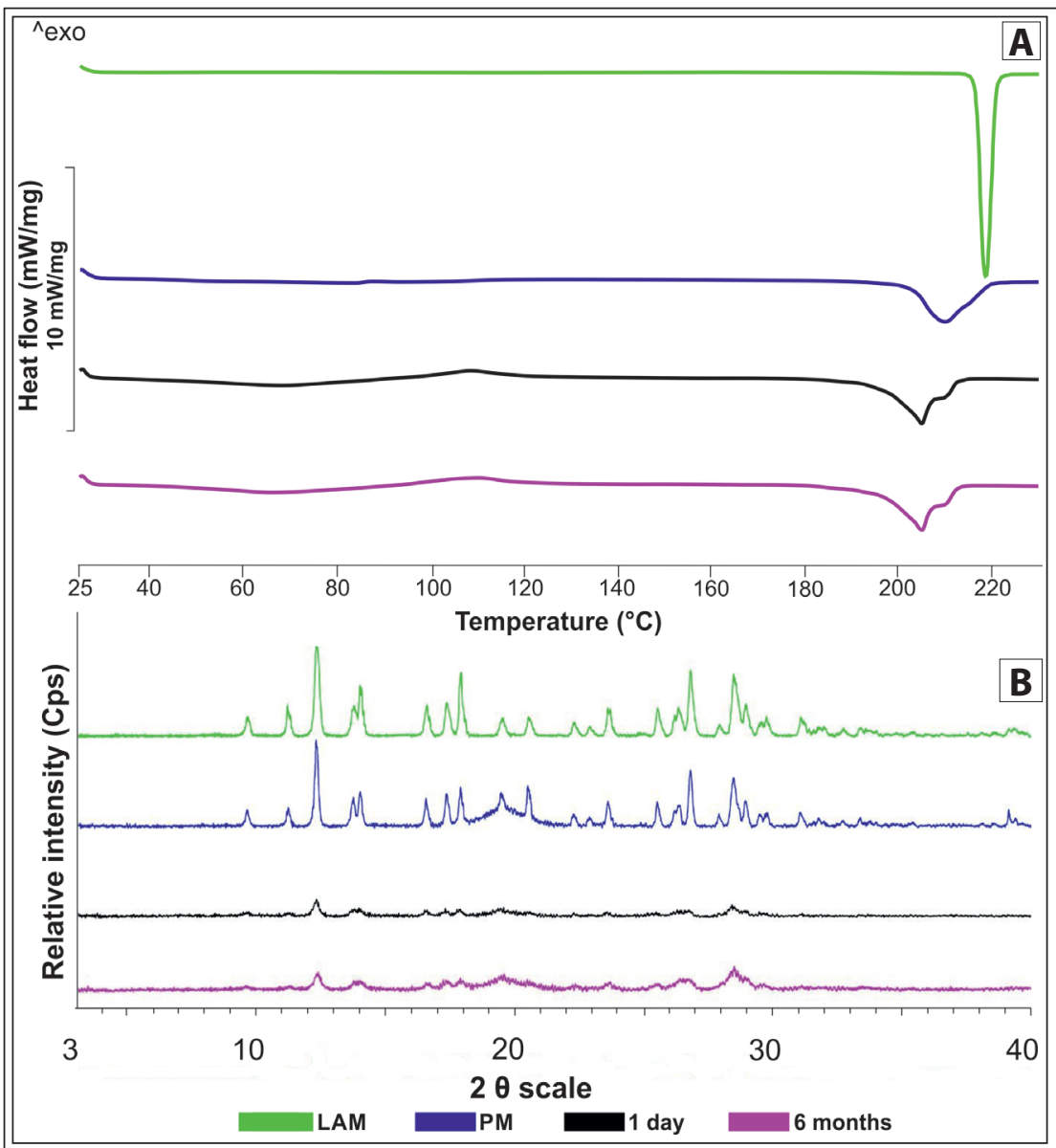

Figure 2 The DSC curves (A) and the XRPD diffractograms (B) of the samples

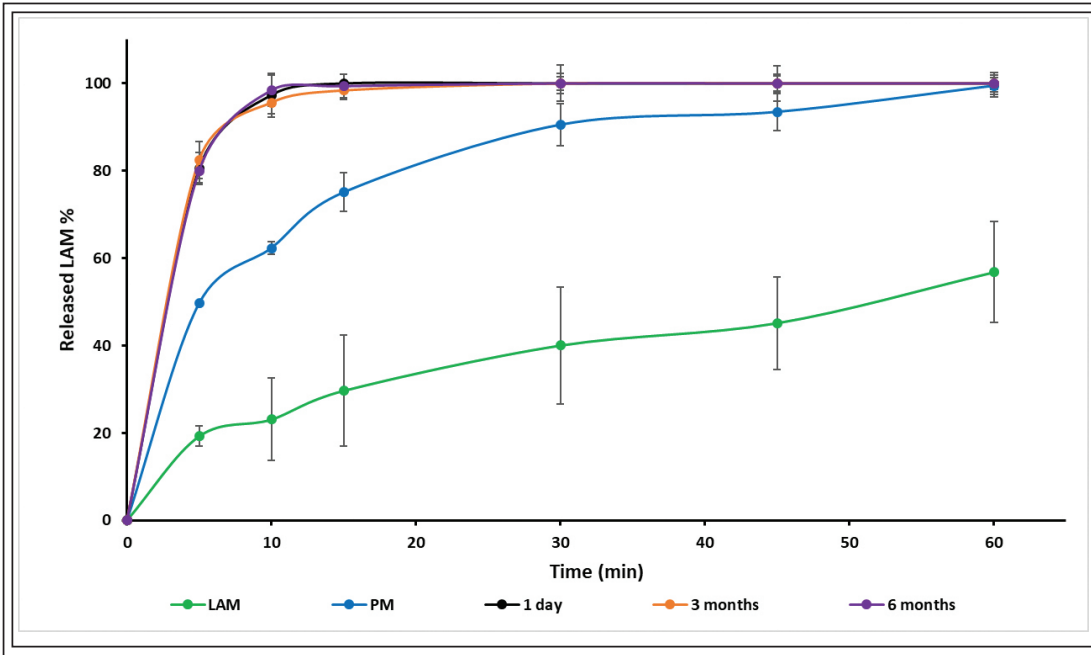

Figure 3 The results of the release study, where LAM is the raw lamotrigine and $P M$ is the Physical Mixture

Table 3 The crystallinity index $\left(X_{c}\right)$ values of the samples

\begin{tabular}{l|c|c}
\hline \multirow{2}{*}{} & \multicolumn{2}{|c}{$\mathbf{X}_{\mathbf{c}}(\%)$} \\
\cline { 2 - 3 } & DSC & XRPD \\
\hline PM & 100 & 100 \\
\hline 1-day & 41.91 & 47.58 \\
\hline 6 months & 47.53 & 48.40 \\
\hline
\end{tabular}

diffractograms, the characteristic $2 \theta^{\circ}$ values of the raw LAM were at 12.57, 19.62, 27.04, 27.19 and 28.92 [13], which can be also seen in the case of the PM. Due to the co-milling with PVA, the crystallinity of the LAM decreased as the intensity of the characteristic peaks decreased in the 1-day sample. After 6 months, this decreased crystallinity was maintained. The crystallinity index was found $47.58 \%$ in the 1-day sample, which value did not change considerably during the storage.

Table 3 shows the crystallinity index values of the samples. The results of the measurement confirmed the DSC results, which means that the LAM became and remained partly amorphous due to the milling effect and the presence of PVA in the tested period. Moreover, the TG results showed no change in mass, which refers that no dehydration or decomposition occurred during the investigation.

\subsection{In vitro release study}

In Figure 3, it can be seen that the LAM from the surface of the polymer released rapidly in a high amount in the case of the NP sample compared to the PM and raw LAM. This rapid release was maintained during the examined period as practically there is no difference between the samples. The rapid dissolution rate was occurred due to the nanosized property of LAM and the presence of PVA.

\section{Conclusion}

To conclude the study, it can be said that the key properties of the NP product did not change considerably. The particle size of the product did not show relevant change during the tested period, while the particle size of LAM in the formulation 
showed an increase with no significant difference. Moreover, the partly amorphous property of LAM was maintained during the stability test and according to the TG results, no dehydration or decomposition occurred, which suggests that the sample did not remove water from its surroundings during storage. The results of the dissolution studies showed a rapid and high amount of the released drug in the examined period. The results predict that PVA could prevent the particles from aggregation and recrystallization. However, further stability investigations are required - according to ICH guidelines -, but it they will be worth carrying out in a final package. According to this study, it is predictable that the product can maintain its quality over a long period and thus to produce a proper effect.

\section{Acknowledgment}

This work was supported by Gedeon Richter Ltd.-GINOP project (2.3.2-15-2016-00060) and The Ministry of Human Capacities, Hungary grant 20391-3/2018/FEKUSTRAT is also acknowledged.

\section{References}

1. H. Kublik, M.T. Vidgren, Nasal delivery systems and their effect on deposition and absorption, Advanced Drug Delivery Reviews. 1998;29:157-177. https://doi. org/10.1016/S0169-409X(97)00067-7.

2. U. Anand, T. Feridooni, R. U., Novel Mucoadhesive Polymers for Nasal Drug Delivery, in: A.D. Sezer (Ed.), Recent Advances in Novel Drug Carrier Systems, InTech, 2012. https://doi.org/10.5772/52560.

3. S.S. Chudiwal, M.H.G. Dehghan, Quality by design approach for development of suspension nasal spray products: a case study on budesonide nasal suspension, Drug Development and Industrial Pharmacy. 2016;42:16431652. https://doi.org/10.3109/03639045.2016.1160108.

4. R.P. Chen, From Nose to Brain: The Promise of Peptide Therapy for Alzheimer's Disease and Other Neurodegenerative Diseases, Journal of Alzheimer's Disease \& Parkinsonism. 2017;07. https://doi.org/10.4172/21610460.1000314.

5. Y.S.R. Elnaggar, S.M. Etman, D.A. Abdelmonsif, O.Y. Abdallah, Intranasal Piperine-Loaded Chitosan Nanoparticles as Brain-Targeted Therapy in Alzheimer's Disease: Optimization, Biological Efficacy, and Potential Toxicity, Journal of Pharmaceutical Sciences. 2015;104:3544-3556. https://doi.org/10.1002/jps.24557.
6. E. Prommer, L. Thompson, Intranasal fentanyl for pain control: current status with a focus on patient considerations, Patient Preference and Adherence. 2011;157. https://doi.org/10.2147/PPA.S7665.

7. R. Narayan, M. Singh, O. Ranjan, Y. Nayak, S. Garg, G.V. Shavi, U.Y. Nayak, Development of risperidone liposomes for brain targeting through intranasal route, Life Sciences. 2016;163:38-45. https://doi.org/10.1016/j. lfs.2016.08.033.

8. A. Mistry, S. Stolnik, L. Illum, Nanoparticles for direct nose-to-brain delivery of drugs, International Journal of Pharmaceutics. 2009;379:146-157. https://doi. org/10.1016/j.ijpharm.2009.06.019.

9. P. Ruenraroengsak, J.M. Cook, A.T. Florence, Nanosystem drug targeting: Facing up to complex realities, Journal of Controlled Release. 2010;141:265-276. https://doi.org/10.1016/j.jconrel.2009.10.032.

10. C. Callens, J. Ceulemans, A. Ludwig, P. Foreman, J.P. Remon, Rheological study on mucoadhesivity of some nasal powder formulations, European Journal of Pharmaceutics and Biopharmaceutics. 2003;55:323-328. https://doi.org/10.1016/S0939-6411(03)00024-9.

11. A. Tanaka, T. Furubayashi, M. Tomisaki, M. Kawakami, S. Kimura, D. Inoue, K. Kusamori, H. Katsumi, T. Sakane, A. Yamamoto, Nasal drug absorption from powder formulations: The effect of three types of hydroxypropyl cellulose (HPC), European Journal of Pharmaceutical Sciences. 2017;96:284-289. https://doi. org/10.1016/j.ejps.2016.09.028.

12. Z.T. Al-Salama, L.J. Scott, Sumatriptan Nasal Powder: A Review in Acute Treatment of Migraine, Drugs. 2016;76:1477-1484. https://doi.org/10.1007/s40265-0160641-9.

13. P. Gieszinger, I. Csóka, E. Pallagi, G. Katona, O. JójártLaczkovich, P. Szabó-Révész, R. Ambrus, Preliminary study of nanonized lamotrigine containing products for nasal powder formulation, Drug Design, Development and Therapy. Volume 2017;11:2453-2466. https:// doi.org/10.2147/DDDT.S138559.

14. P. Gieszinger, I. Tomuta, T. Casian, Cs. Bartos, P. Szabó-Révész, R. Ambrus, Definition and validation of the Design Space for co-milled nasal powder containing nanosized lamotrigine, Drug Development and Industrial Pharmacy. 2018;44:1622-1630. https://doi.org/1 0.1080/03639045.2018.1483388.

15. R. Ambrus, P. Gieszinger, R. Gáspár, A. SztojkovIvanov, E. Ducza, Á. Márki, T. Janáky, F. Tömösi, G. Kecskeméti, P. Szabó-Révész, C. Bartos, Investigation of the Absorption of Nanosized lamotrigine Containing Nasal Powder via the Nasal Cavity, Molecules. 2020;25:1065. https://doi.org/10.3390/molecules25051065.

16. $\mathrm{CPMP} / \mathrm{ICH} / 380 / 95$, Stability testing guidelines: Stability Testing of New Drug Substances and Products, (1993). http://www.pharma.gally.ch/ich/q1a038095en. pdf (accessed March 11, 2020).

17. M.D. Abràmoff, Image Processing with ImageJ, (n.d.) 7. 